\title{
Urothelial-Type Adenocarcinoma of the Prostate Mimicking Metastatic Colorectal Adenocarcinoma
}

\author{
Brian P. Adley, Kelly Maxwell, Daniel P. Dalton, Ximing J. Yang \\ Departments of Pathology (BPA, XJY) and Urology (KM, DPD, XJY) Northwestern University, \\ Feinberg School of Medicine, Chicago, Illinois, USA
}

\begin{abstract}
Adenocarcinoma arising in urinary bladder or prostatic urethra is uncommon. When they occur, the tumor can be mistaken for metastatic lesions, especially from the colon. Here we report the fifth case of a primary urothelial-type adenocarcinoma arising in the prostate which showed enteric differentiation. The patient was a 55 year-old male whose prostatic needle core biopsy showed a high grade adenocarcinoma which was initially thought to be metastatic colon cancer. A follow-up colonoscopy was unremarkable. Subsequent prostatectomy revealed a high grade adenocarcinoma which was positive for cytokeratins 7 and 20, carcinoembryonic antigen, CDX2, and high molecular weight cytokeratin, and negative for prostate specific antigen, prostate specific acid phosphatase and AMACR. A diagnosis of urothelial-type adenocarcinoma of the prostate was rendered. We review the literature regarding this entity, and discuss the differential diagnosis, emphasizing utility of immunohistochemistry in making the diagnosis. Finally, we speculate on the behavior of these rare tumors.
\end{abstract}

Key words: prostate; urothelial-type adenocarcinoma; pathology

Int Braz J Urol. 2006; 32: 681-8

\section{INTRODUCTION}

Urothelial-type adenocarcinomas with mucinous/enteric differentiation arising in the prostatic gland is exceedingly rare, with only 4 case reports found in the literature. Tran \& Epstein (1) described 2 cases of mucinous adenocarcinoma of urethral type which occurred in the setting of urethritis glandularis and associated adenocarcinoma in situ. Curtis et al. (2) described 2 additional cases in which both were centered around the prostatic urethra but were not associated with urethritis glandularis. The authors discussed the utility of immunohistochemistry in distinguishing these tumors from other histologic mimickers including conventional prostatic adenocarcinoma and metastatic colorectal adenocarcinoma (2).

Here we report a case of rare urothelial-type adenocarcinoma with enteric differentiation arising in the prostate. The initial biopsy was suggestive of metastatic colorectal carcinoma to the prostate. We describe the clinicopathologic features and discuss the importance of immunohistochemistry in differentiating these two entities.

\section{CASE REPORT}

A 55 year old male presented to the urology clinic at our institution with a serum prostate-specific antigen (PSA) of $10.0 \mathrm{ng} / \mathrm{mL}$ and abnormal digital 
rectal examination. Past medical history included hypertension and gout. The patient denied alcohol intake and quit smoking 10 years prior. The patient subsequently underwent a prostatic needle core biopsy which showed prostatic acinar adenocarcinoma, Gleason's grade $3+3$ in 2 of 4 cores (30\% of examined tissue) from the right lobe. In addition, the presence of high grade adenocarcinoma morphologically similar to colonic adenocarcinoma was observed in the left lobe. Based on the pathologic suspicion of metastatic colon cancer, a complete work-up was conducted to locate a primary colon cancer. An MRI of the pelvis showed diffuse involvement of the peripheral zone of left prostate gland (apex to base) with extension across the midline and possible extension to the left seminal vesicle. The patient subsequently had an unremarkable colonoscopy and negative colonic biopsy. A bone scan failed to reveal any metastatic tumor at that time. The patient underwent a retropubic radical prostatectomy with pelvic lymph node dissection for the prostatic acinar adenocarcinoma.

\section{PATHOLOGIC FINDINGS}

\section{Gross}

The radical prostatectomy specimen weighed 92 grams and measured $8.0 \times 7.0 \times 4.5 \mathrm{~cm}$. The right seminal vesicle measured $2.5 \times 2.0 \times 1.4 \mathrm{~cm}$, and the left seminal vesicle measured $1.0 \times 1.0 \times 0.7 \mathrm{~cm}$. The external surface of the prostate was tan with a shaggy appearance. Serially sectioning from apex to base revealed a large, tan-white, firm and focally necrotic, infiltrative mass lesion measuring $3.5 \times 3.0 \times 2.0 \mathrm{~cm}$, replacing almost the entire left lobe. This mass grossly extended into the capsule and the left seminal vesicle. The remaining cut surface of the prostate had a tan nodular appearance without additional discernable tumors.

\section{Microscopic}

Hematoxylin and eosin (HE) stained sections from both the left needle core biopsy and prostatectomy specimens showed a high grade adenocarcinoma with glandular and cribriform architecture infiltrating the prostatic parenchyma (Figure-1). Abundant "dirty-type" necrosis within malignant glandular structures, characteristically seen in colonic-type adenocarcinoma, was identified (Figure-2). The tumors cells were columnar to polygonal with high nuclear to cytoplasmic ratios, marked pleomorphism, and prominent nucleoli

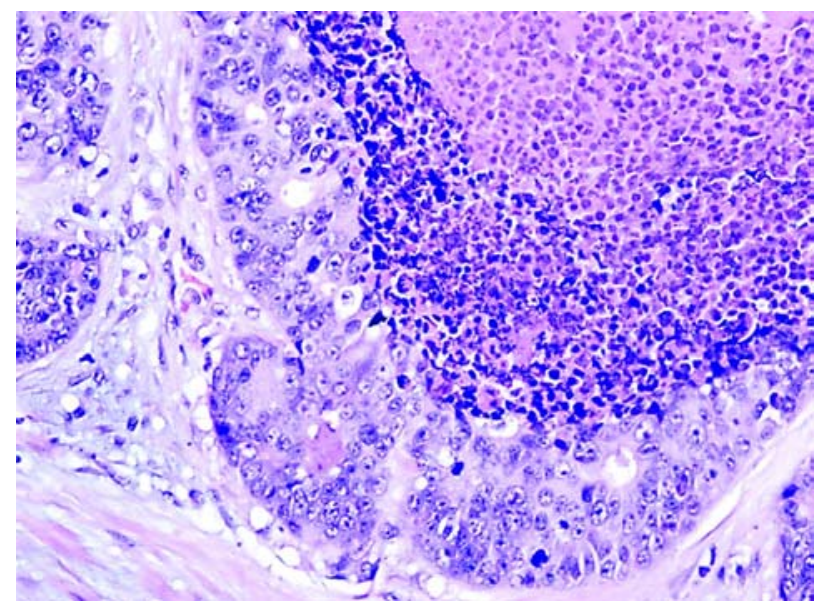

Figure 1-Prostatectomy with high-grade adenocarcinoma with glandular and cribriform architecture (HE, X400).

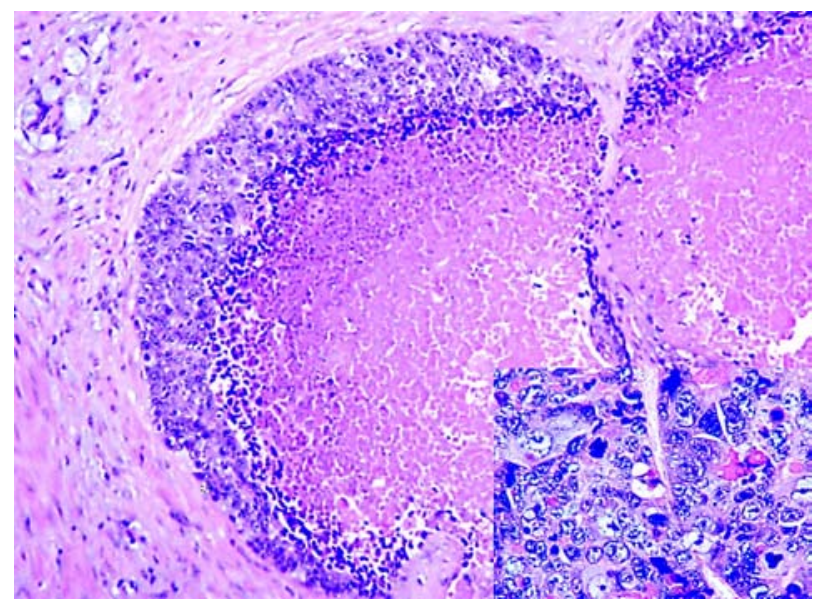

Figure 2 - Abundant "dirty-type" necrosis characteristically seen in colonic-type adenocarcinoma was identified within many malignant glands (HE, X200). Note the small focus of conventional prostatic acinar adenocarcinoma in upper left corner. The tumor cells were columnar to polygonal with high nuclear to cytoplasmic ratios, marked pleomorphism, prominent nucleoli and numerous mitosis (Inset, HE, X400). 
(Figure-2, inset). They displayed abundant mitotic activity and apoptosis. No intracellular or extracellular mucin was identified. The morphology seen on the H\&E stains was very reminiscent of a high grade enteric-type adenocarcinoma. The tumor was predominantly present on the left side, with extensive extraprostatic extension and focally positive margins. Perineural as well as vascular invasion was also identified. The tumor involved approximately $90 \%$ of the left lobe and $10 \%$ of the right lobe of the gland. It also extended into both seminal vesicles microscopically. Both left and right pelvic lymph nodes sampled were negative for metastatic tumor.

A second population of tumor cells was present in both the right side core biopsy and prostatectomy specimens, mostly in the right lobe. It consisted of infiltrating acinar structures composed of uniform cuboidal cells with prominent nucleoli, morphologically characteristic of conventional prostatic acinar adenocarcinoma, Gleason's score 4+3 (Figure-3). The tumor was a minor component in the prostatectomy specimen occupying less than $5 \%$ of the total gland volume and was in close proximity to the enteric type adenocarcinoma.

Immunohistochemistry for cytokeratin 20 (CK20), prostate-specific antigen (PSA), and CDX2 were performed on the right-sided core biopsy containing the high grade adenocarcinoma reminiscent of colonic adenocarcinoma. The tumor was diffusely and strongly positive for CK20 and CDX2 and negative for PSA. This led to the suspicion of metastatic poorly differentiated adenocarcinoma consistent with colonic primary on prostatic biopsy specimen.

Furthermore, a panel of immunohistochemical stains for cytokeratin 7 and 20, PSA, PSAP, thrombomodulin, alpha methylacyl CoA racemase (AMACR), CDX2, monoclonal carcinoembryonic antigen (mCEA), CD31, human chorionic gonadotropin (HCG), alpha-fetoprotein (AFP), placental alkaline phosphatase (PLAP), and highmolecular weight keratin (HMWK) were performed on sections from the prostatectomy specimen. The high grade adenocarcinoma showed strong diffuse expression of CK20, CDX2, and HMWK as well as

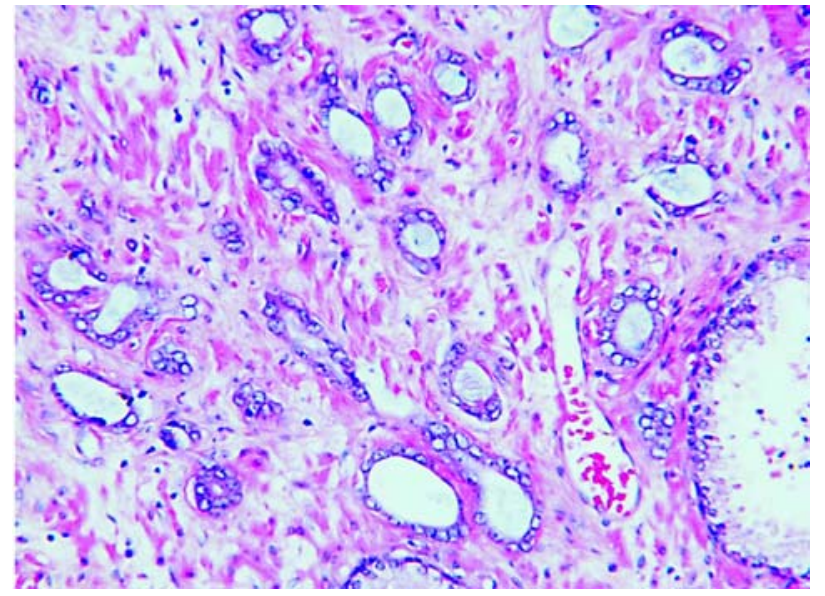

Figure 3 - Infiltrating acinar structures composed of uniform cuboidal cells with prominent nucleoli, morphologically consistent with prostatic acinar adenocarcinoma (HE, X200).

focal expression of CK7 and mCEA. The tumor was completely negative for PSA, PSAP, thrombomodulin, AMACR, CD31, AFP, and PLAP. Less than $1 \%$ of cells were positive for HCG. The immunostaining results are summarized in Table-1 and partially illustrated in Figure- 4 and 5.

\section{COMMENTS}

Within the prostate gland, urothelium lines primarily the prostatic urethra but may extend to portions of major prostatic ducts. Even prostatic acini can undergo urothelial (transitional) metaplasia. Theoretically, any lesion which is known to occur elsewhere in the urothelial tract may occur in the prostate gland, and urothelial lesions such as inverted papilloma and urothelial carcinoma have all been described in the prostate (3-5). Adenocarcinoma, a tumor usually occurring in the bladder or urethra, can assume patterns similar to colonic adenocarcinoma including the typical enteric pattern, signet-ring pattern, and mucinous pattern (6,7). It is suggested that adenocarcinoma of bladder or urethra usually arises in precursor lesions like urethritis glandularis (1) or periurethral structures such as Cowper's glands. Urothelial-type adenocarcinoma arising in the prostate is exceedingly rare, with only four previous cases reported in the literature. 


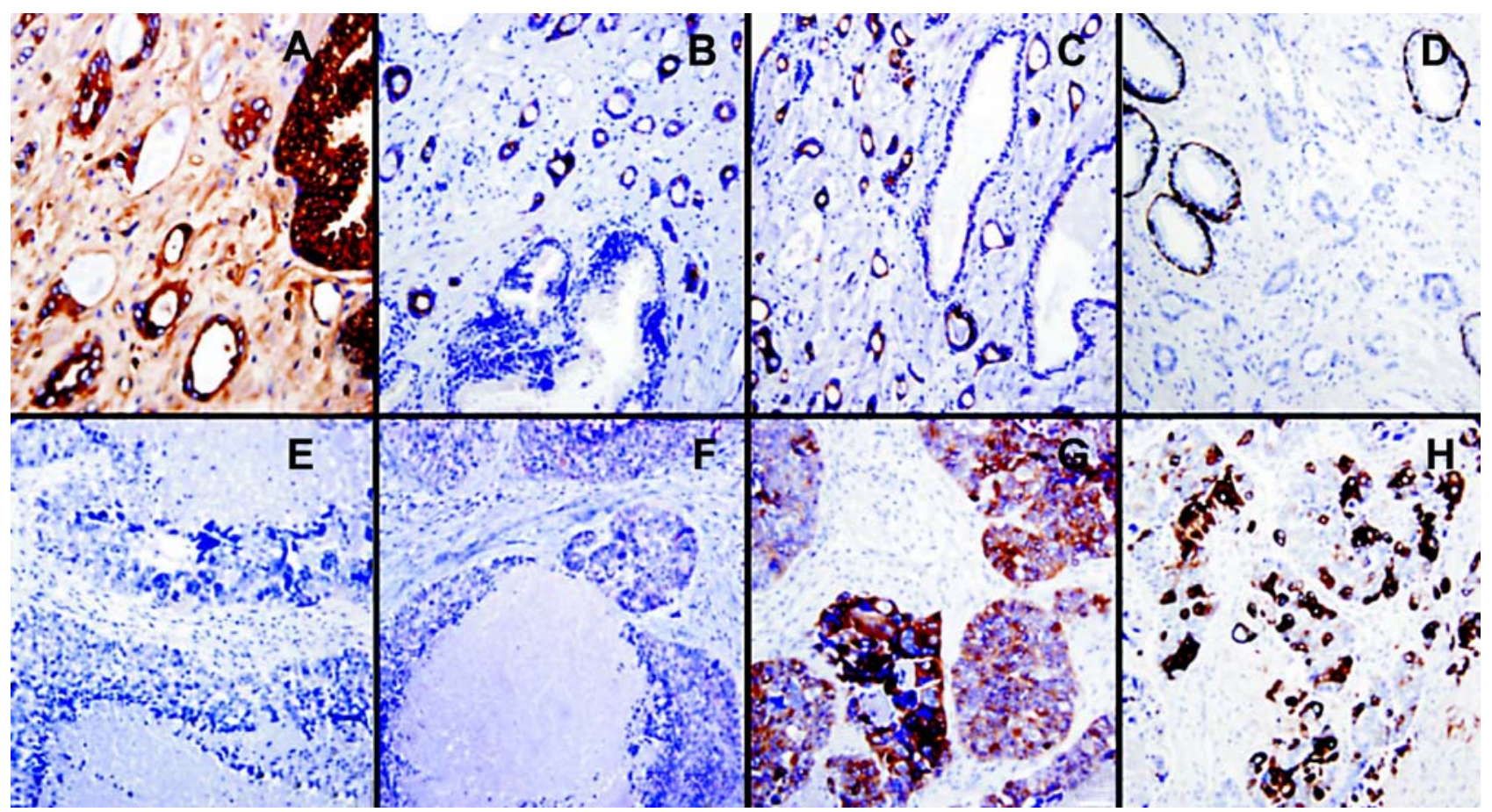

Figure 4 - Immunohistochemical results in conventional prostatic acinar adenocarcinoma $(A-D)$ and urothelial-type adenocarcinoma in prostatectomy specimen. The conventional acinar adenocarcinoma was positive for PSA (A), AMACR (B), and CK7 (C), but negative for HMWCK $(D)$. The urothelial-type adenocarcinoma was negative for PSA $(E)$ and AMACR $(F)$ but positive for $C K 7(G)$ and $H M W C K$ (H), Immunolabelling, X400.

The differential diagnosis for urothelial-type adenocarcinoma of the prostate includes prostatic mucinous adenocarcinoma, prostatic ductal adenocarcinoma, and metastatic colonic adenocarcinoma. Mucinous adenocarcinoma of the prostate typically has cribriform glands with bland cytology characteristic of conventional prostatic acinar adenocarcinoma. These tumors are typically

Table 1 - Summary of immunohistochemical results for urothelial-type adenocarcinoma and prostatic acinar adenocarcinoma.

\begin{tabular}{llcll}
\hline Antibody & Company & Dilution & $\begin{array}{c}\text { Urothelial-Type } \\
\text { Adenocarcinoma }\end{array}$ & $\begin{array}{c}\text { Prostatic Acinar } \\
\text { Adenocarcinoma }\end{array}$ \\
\hline AMACR & Zeta Co. & $1: 100$ & negative & positive \\
CDX2 & Biogenex & $1: 20$ & positive (weakly) & negative \\
CK7 & Dako & $1: 100$ & positive (focally) & positive (focal) \\
CK20 & Dako & $1: 25$ & positive & positive (focal) \\
HMWCK & Dako & $1: 50$ & positive & negative \\
CEA & Dako & $1: 100$ & positive & negative \\
HCG & Dako & $1: 25$ & positive (1\% cells) & negative \\
PSAP & Dako & $1: 50$ & negative & positive \\
PSA & Dako & $1: 1800$ & negative & positive \\
\hline
\end{tabular}

AMACR = alpha methylacyl CoA racemase; $C K=$ cytokeratin $; H M W C=$ high molecular weight cytokeratin; $C E A=$ carcinoembryonic antigen $; H C G=$ human chorionic gonadotropin; $P S A P=$ prostate-specific acid phosphatase $;$ PSA = prostate-specific antigen . 


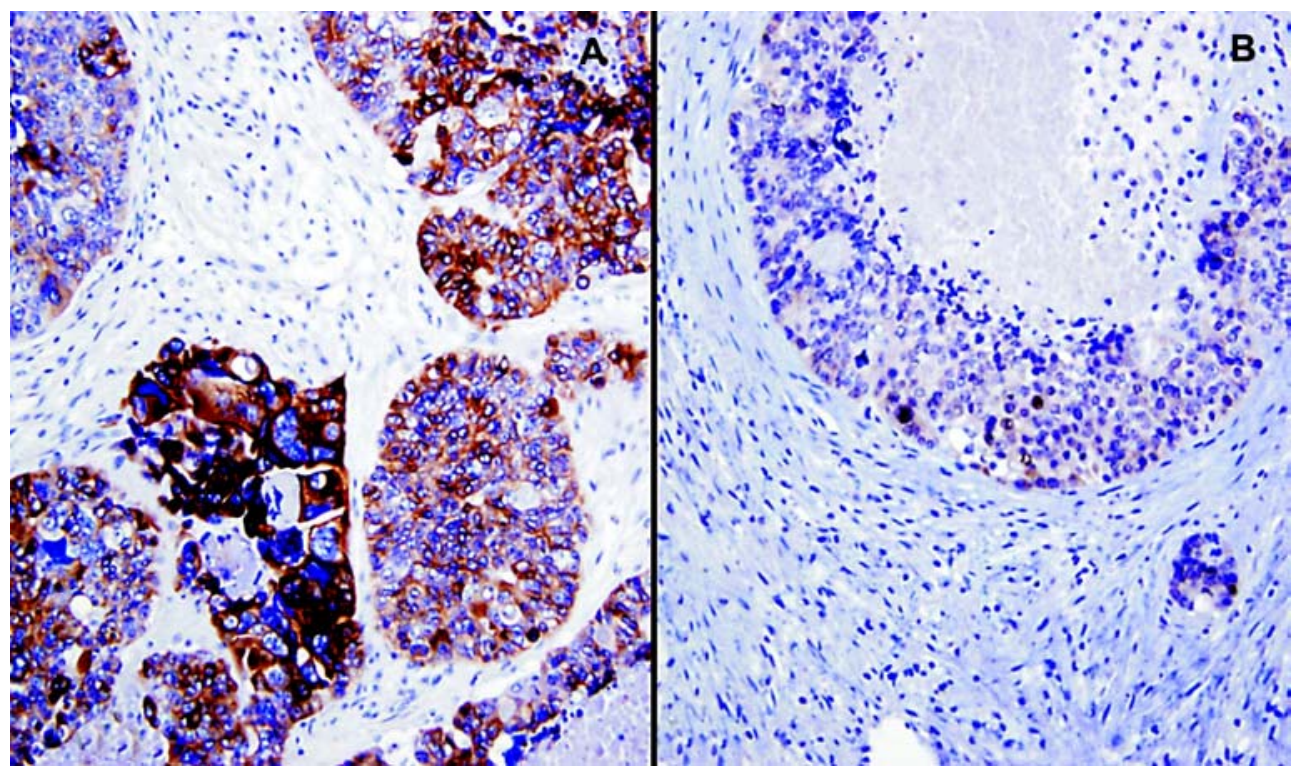

Figure 5 - Similar to colorectal adenocarcinoma, the urothelial-type adenocarcinoma was diffusely positive for CK20 (A) and CDX2 (B), Immunolabelling, X400.

intermingled with conventional acinar adenocarcinoma, and usually classified as Gleason score $3+4$. The epithelium may float in mucin and the cells express PSA and PSAP (8). Ductal adenocarcinomas of the prostate may very closely resemble urothelial type adenocarcinoma but are also positive for PSA and PSAP (9).

Perhaps the most challenging differential diagnosis is that of metastatic colorectal adenocarcinoma, which can look almost identical to urothelial-type adenocarcinoma. Immunohistochemistry can be helpful in these situations as we previously reported (10). Cytokeratin 7 (CK7) is typically positive in urothelial carcinoma but negative in colorectal carcinoma (10), although both tumors are positive for cytokeratin 20. In this case, tumor cells were focally positive for CK7. Villin, reported to be positive in colonic adenocarcinoma and negative in urothelial carcinoma with glandular differentiation, has been shown to be expressed in enteric-type urothelial adenocarcinoma (11). High molecular weight keratins, including CK 1, 5, 10 and 14, have been shown to be highly expressed in urothelial carcinomas and only rarely expressed in colonic and prostatic adenocarcinomas (12). It has also been reported that HCG producing tumor cells can be seen in urothelial carcinoma.

AMACR was first identified as a prostate cancer marker (13). Further studies showed its expression in the majority of colonic adenocarcinomas (14). In addition, AMACR is also positive in prostatic mucinous adenocarcinoma and ductal adenocarcinoma, although the reactivity may be reduced focally. AMACR is typically negative or weakly positive in urothelial carcinoma and positive in a small percentage of adenocarcinomas of the bladder (15). The negative AMACR stain in this high grade urothelial-type adenocarcinoma supports its urothelial differentiation and is inconsistent with prostatic ductal adenocarcinoma or colonic adenocarcinoma. In the case reported here, the finding of a small subset of HCG positive cells also supports the urothelial origin or differentiation of this tumor.

Curtis et al. (2) proposed that a panel of antibodies to PSA, PSAP, CK7, CK20 and HMWK would be useful in differentiating urothelial-type adenocarcinoma, metastatic colorectal adenocarcinoma, and conventional prostatic acinar adenocarcinoma. Urothelial-type adenocarcinomas should be positive for HMWK and CK7 and may be 
positive for CK20, but should not express PSA and PSAP. Colorectal carcinoma should be positive for CK20 but negative for all other markers. Prostatic acinar or ductal adenocarcinoma should express PSA and PSAP but be negative for all other markers. Our tumor strongly expressed CK20 and CEA, and was focally positive for CDX2. This profile may suggest a colorectal primary. However, our tumor also expressed HMWK and CK7, which is not typical of colon cancer. More importantly, the patient had a completely negative workup for primary colon cancer. Hence we feel that our case is most consistent with urothelial-type adenocarcinoma of the prostate gland showing a degree of enteric differentiation. Although our tumor did not contain mucin, it had other characteristics commonly seen in colorectal carcinoma including extensive dirty necrosis and a focal cribriform growth pattern.

The etiology of this malignant tumor is unknown. Although urethritis glandularis has been proposed to be a precursor lesion for urothelial-type adenocarcinoma, our patient did not have evidence of urethritis glandularis. In fact, the sampled prostatic urethral mucosa was histologically unremarkable. One possibility is that the tumor arose from a prostatic duct, which may be lined by urothelial-type mucosa. Of interest, our patient also had a small focus of conventional acinar-type prostatic adenocarcinoma, which was also described in two of the four previous case reports. Therefore, an alternative possibility is that this tumor may be derived from a stem cell which underwent biphasic differentiation to urothelial-type adenocarcinoma and conventional prostatic acinar adenocarcinoma.

Due to the rarity of these tumors, it is difficult to predict their behavior. One previously reported patient died of disease with liver metastasis within one year of initial diagnosis (2). Another patient experienced local recurrence 4 years status-post simple prostatectomy (1). The two other patients showed no evidence of disease one year ${ }^{1}$ and 16 months (2) status-post radical prostatectomy. Our patient developed both local recurrence as well as multiple metastases to lung, liver, and bone within 4 months of prostatectomy. It was observed that the metastatic tumors of this patient were morphologically and immunohistochemically identical to the urothelial-type adenocarcinoma of the prostate. Therefore, it is important to note that these tumors may behave in an aggressive manner.

In conclusion, we have described a rare high grade urothelial-type adenocarcinoma arising in the prostate. This primary prostatic tumor, mimicking metastatic colorectal adenocarcinoma due to its striking morphologic resemblance as well as overlapping immunohistochemical phenotype, should be recognized for appropriate clinical management. Our study supports the previous notion that high molecular weight keratin may be the key marker for differentiating these tumors from colorectal metastasis.

\section{CONFLICT OF INTEREST}

None declared.

\section{REFERENCES}

1. Tran KP, Epstein JI: Mucinous adenocarcinoma of urinary bladder type arising from the prostatic urethra. Distinction from mucinous adenocarcinoma of the prostate. Am J Surg Pathol. 1996; 20: 1346-50.

2. Curtis MW, Evans AJ, Srigley JR: Mucin-producing urothelial-type adenocarcinoma of prostate: report of two cases of a rare and diagnostically challenging entity. Mod Pathol. 2005; 18: 585-90.

3. Malpica A, Ro JY, Troncoso P, Ordonez NG, Amin MB, Ayala AG: Nephrogenic adenoma of the prostatic urethra involving the prostate gland: a clinicopathologic and immunohistochemical study of eight cases. Hum Pathol. 1994; 25: 390-5.

4. Nicolaisen GS, Williams RD: Primary transitional cell carcinoma of prostate. Urology. 1984; 24: 544-9.

5. Sawczuk I, Tannenbaum M, Olsson CA, deVere White R: Primary transitional cell carcinoma of prostatic periurethral ducts. Urology. 1985; 25: 339-43.

6. Grignon DJ, Ro JY, Ayala AG, Johnson DE, Ordonez NG: Primary adenocarcinoma of the urinary bladder. A clinicopathologic analysis of 72 cases. Cancer. 1991; 67: 2165-72.

7. Wang HL, Lu DW, Yerian LM, Alsikafi N, Steinberg 
G, Hart J, et al.: Immunohistochemical distinction between primary adenocarcinoma of the bladder and secondary colorectal adenocarcinoma. Am J Surg Pathol. 2001; 25: 1380-7.

8. Epstein JI, Lieberman PH: Mucinous adenocarcinoma of the prostate gland. Am J Surg Pathol. 1985; 9: 299308.

9. Christensen WN, Steinberg G, Walsh PC, Epstein JI: Prostatic duct adenocarcinoma. Findings at radical prostatectomy. Cancer. 1991; 67: 2118-24.

10. Wang NP, Zee S, Zarbo RJ, Bacchi CE, Gown AM: Coordinate expression of cytokeratins 7 and 20 defines unique subsets of carcinomas. Appl Immunohistochem 1995; 3: 99-107.

11. Tamboli P, Mohsin SK, Hailemariam S, Amin MB: Colonic adenocarcinoma metastatic to the urinary tract versus primary tumors of the urinary tract with glandular differentiation: a report of 7 cases and investigation using a limited immunohistochemical panel. Arch Pathol Lab Med. 2002; 126: 1057-63.
12. Yang XJ, Lecksell K, Gaudin P, Epstein JI: Rare expression of high-molecular-weight cytokeratin in adenocarcinoma of the prostate gland: a study of 100 cases of metastatic and locally advanced prostate cancer. Am J Surg Pathol. 1999; 23: 147-52.

13. Xu J, Stolk JA, Zhang X, Silva SJ, Houghton RL, Matsumura M, et al.: Identification of differentially expressed genes in human prostate cancer using subtraction and microarray. Cancer Res. 2000; 60: 1677-82.

14. Jiang Z, Fanger GR, Banner BF, Woda BA, Algate P, Dresser K, et al.: A dietary enzyme: alpha-methylacyl$\mathrm{CoA}$ racemase/P504S is overexpressed in colon carcinoma. Cancer Detect Prev. 2003; 27: 422-6.

15. Suh N, Yang XJ, Tretiakova MS, Humphrey PA, Wang HL: Value of CDX2, villin, and alpha-methylacyl coenzyme A racemase immunostains in the distinction between primary adenocarcinoma of the bladder and secondary colorectal adenocarcinoma. Mod Pathol. 2005; 18: 1217-22.

\author{
Correspondence address: \\ Dr. Brian P. Adley \\ Department of Pathology, Feinberg 7-325 \\ Northwestern Univ, Feinberg Sch Med \\ 251 E. Huron Street \\ Chicago, Illinois, 60611, USA \\ Fax: + 1312 926-3127 \\ E-mail: b-adley@northwestern.edu
}

\section{EDITORIAL COMMENT}

This is a very well written paper on a very rare tumor. Adenocarcinoma of the urinary tract may correspond to 3 possibilities: glandular differentiation of a conventional urothelial carcinoma (more frequently), secondary involvement (more frequently from the prostate or colorectal region), and primary (more rarely). Clinical work-up as well as morphology characteristics and immunohistochemistry help in the differential diagnosis. 


\section{Urothelial-Type Adenocarcinoma of the Prostate}

Primary adenocarcinomas may also arise from the urethra or distal prostatic ducts. In this case the differential diagnosis is much more difficult because the great majority of the cases are conventional adenocarcinomas of the prostate and the pathologist is not aware of the possibility of dealing with a so rare condition. The differential must be done with a thorough clinical work-up to exclude adenocarcinoma of the rectum or colon and, on morphology, only by immunohistochemistry.

Only 4 cases were previously published of a primary urothelial-type adenocarcinoma of the prostate. The final diagnosis is mainly based on immunohistochemistry.

Dr. Athanase Billis

Full-Professor of Pathology State University of Campinas, Unicamp

Campinas, São Paulo, Brazi E-mail:athanase@fcm.unicamp.br 